\title{
Singular Value Inequalities for Compact Normal Operators
}

\author{
Wasim Audeh \\ Department of Basic Sciences, Petra University, Amman, Jordan \\ Email: waudeh@uop.edu.jo
}

Received September 25, 2013; revised October 28, 2013; accepted November 7, 2013

Copyright (c) 2013 Wasim Audeh. This is an open access article distributed under the Creative Commons Attribution License, which permits unrestricted use, distribution, and reproduction in any medium, provided the original work is properly cited.

\section{ABSTRACT}

We give singular value inequality to compact normal operators, which states that if $A$ is compact normal operator on a complex separable Hilbert space, where $A=A_{1}+i A_{2}$ is the cartesian decomposition of $A$, then $\frac{1}{\sqrt{2}} s_{j}\left(A_{1}+A_{2}\right) \leq s_{j}(A) \leq s_{j}\left(\left|A_{1}\right|+\left|A_{2}\right|\right)$ for $j=1,2, \cdots$ Moreover, we give inequality which asserts that if $A$ is compact normal operator, then $\sqrt{2} s_{j}\left(A_{1}+A_{2}\right) \leq s_{j}\left(A+i A^{*}\right) \leq 2 s_{j}\left(A_{1}+A_{2}\right)$ for $j=1,2, \cdots$ Several inequalities will be proved.

Keywords: Compact Operator; Inequality; Normal Operator; Self-Adjoint Operator; Singular Value

\section{Introduction}

Let $B(H)$ denote the space of all bounded linear operators on a complex separable Hilbert space $\mathrm{H}$, and let $K(H)$ denote the two-sided ideal of compact operators in $B(H)$. For $T \in K(H)$, the singular values of $T$, denoted by $s_{1}(T), s_{2}(T), \cdots$ are the eigenvalues of the positive operator $|\mathrm{T}|=\left(T^{*} T\right)^{1 / 2}$ as $s_{1}(T) \geq s_{2}(T) \geq \cdots$ and repeated according to multiplicity. Note that $s_{j}(T)=s_{j}\left(T^{*}\right)=s_{j}|T|$ for $j=1,2, \cdots$ It follows Weyl's monotonicity principle (see, e.g., [1, p. 63] or [2, p. 26]) that if $S, T \in K(H)$ are positive and $S \leq T$, then $s_{j}(S) \leq s_{j}(T)$ for $j=1,2, \cdots$ The singular values of $S \oplus T$ and $\left[\begin{array}{ll}0 & T \\ S & 0\end{array}\right]$ are the same, and they consist of those of $S$ together with those of $T$. Here, we use the direct sum notation $S \oplus T$ for the blockdiagonal operator $\left[\begin{array}{cc}S & 0 \\ 0 & T\end{array}\right]$ defined on $H \oplus H$.

The Jordan decomposition for self-adjoint operators asserts that every self-adjoint operator can be expressed as the difference of two positive operators. In fact, if $A \in B(H)$ is self-adjoint, then $A=A^{+}-A^{-}$, where
$A^{+}$and $A^{-}$are the positive operators given by

$A^{+}=\frac{|A|+A}{2}$ and $A^{-}=\frac{|A|-A}{2}$, see [1].

Let $A$ be any operator, we can write $A$ in the form $A=A_{1}+i A_{2}$, where $A_{1}=\frac{A+A^{*}}{2}$ and $A_{2}=\frac{A-A^{*}}{2 i}$ are self-adjoint operators, this is called the Cartesian decomposition of the operator $A$. If $A$ is normal, then $A_{1} A_{2}=A_{2} A_{1}$.

Audeh and Kittaneh have proved in [3] that if $A, B, C \in K(H)$ such that $\left[\begin{array}{cc}A & B \\ B^{*} & C\end{array}\right] \geq 0$, then

$$
s_{j}(B) \leq s_{j}(A \oplus C)
$$

for $j=1,2, \cdots$

Also, Audeh and Kittaneh have proved in [3] that if $A, B \in K(H)$ such that $A$ is self-adjoint, $B \geq 0$, and $\pm A \leq B$, then

$$
2 s_{j}(A) \leq s_{j}((B+A) \oplus(B-A))
$$

for $j=1,2, \cdots$

In addition to this, Audeh and Kittaneh have proved in [3] that if $A, B \in K(H)$ be self-adjoint operators, then

$$
s_{j}(A+B) \leq s_{j}\left(\left(A^{+}+B^{+}\right) \oplus\left(A^{-}+B^{-}\right)\right)
$$

for $j=1,2, \cdots$

Zhan has proved in [4] that if $A, B \in K(H)$ are posi- 
tive, then

$$
s_{j}(A-B) \leq s_{j}(A \oplus B)
$$

for $j=1,2, \cdots$ Moreover, it has proved in [3] that (1.3) is a generalization of (1.4).

Hirzallah and Kittaneh have proved in [5] that if $A, B \in K(H)$, then

$$
s_{j}(A+B) \leq 2 s_{j}(A \oplus B)
$$

In this paper, we will give singular value inequalities for normal operators:

Let $A$ be normal operator in $K(H)$. Then

$$
\frac{1}{\sqrt{2}} s_{j}\left(A_{1}+A_{2}\right) \leq s_{j}(A) \leq s_{j}\left(\left|A_{1}\right|+\left|A_{2}\right|\right)
$$

for $j=1,2, \cdots$

We will give singular value inequality to the normal operator $A+i A^{*}$, where $A$ is normal:

Let $A$ be normal operator in $K(H)$. Then

$$
\sqrt{2} s_{j}\left(A_{1}+A_{2}\right) \leq s_{j}\left(A+i A^{*}\right) \leq 2 s_{j}\left(A_{1}+A_{2}\right)
$$

for $j=1,2, \cdots$

\section{Main Results}

We will begin by presenting the following theorem for complex numbers

Theorem 2.1. Let $x=a+i b$ be complex number. Then

$$
\frac{1}{\sqrt{2}}|a+b| \leq|x| \leq|a|+|b|
$$

Also,

$$
\frac{1}{\sqrt{2}}|a-b| \leq|x| \leq|a|+|b|
$$

Proof. The right hand side of the inequalities is well known. To prove the left hand side,

$$
\frac{1}{\sqrt{2}}|a+b|=\frac{1}{\sqrt{2}} \sqrt{(a+b)^{2}}=\frac{1}{\sqrt{2}} \sqrt{a^{2}+2 a b+b^{2}} \leq \frac{1}{\sqrt{2}} \sqrt{a^{2}+a^{2}+b^{2}+b^{2}}=\frac{1}{\sqrt{2}} \sqrt{2 a^{2}+2 b^{2}}=\sqrt{a^{2}+b^{2}}=|x|
$$

Moreover, $\frac{1}{\sqrt{2}}|a-b|=\frac{1}{\sqrt{2}} \sqrt{(a-b)^{2}}=\frac{1}{\sqrt{2}} \sqrt{a^{2}-2 a b+b^{2}} \leq \sqrt{a^{2}+b^{2}}=|x|$.

Now, we will present operator version of Theorem 2.1, inequality (2.1).

Theorem 2.2. Let $A$ be normal operator in $K(H)$, where $A=A_{1}+i A_{2}$ be the Cartesian decomposition of $A$. Then

$$
\frac{1}{\sqrt{2}} s_{j}\left(A_{1}+A_{2}\right) \leq s_{j}(A) \leq s_{j}\left(\left|A_{1}\right|+\left|A_{2}\right|\right)
$$

for $j=1,2, \cdots$

Proof. Let $A=A_{1}+i A_{2}$ be the Cartesian decomposition of the normal operator $A$, which implies that $A_{1} A_{2}=A_{2} A_{1}$. Now, $A^{*} A=A_{1}^{2}+A_{2}^{2}$, it follows that $|A|=\sqrt{A^{*} A}=\sqrt{A_{1}^{2}+A_{2}^{2}}$. In fact $s_{j}(A)=s_{j}(|A|)$ for $j=1,2, \cdots$ By using Weyl's monotonicity principle [1] and the inequality $\sqrt{A_{1}^{2}+A_{2}^{2}} \leq\left|A_{1}\right|+\left|A_{2}\right|$, we get the right hand side of the theorem. To prove the left hand side of the inequality, we will use the inequality which is well known for commuting self-adjoint operators and it asserts that

$$
0 \leq\left(A_{1}+A_{2}\right)^{2} \leq 2\left(A_{1}^{2}+A_{2}^{2}\right)
$$

This implies that

$$
\left|A_{1}+A_{2}\right| \leq \sqrt{2\left(A_{1}^{2}+A_{2}^{2}\right)}
$$

But it is known that $s_{j}\left(A_{1}+A_{2}\right)=s_{j}\left(\left|A_{1}+A_{2}\right|\right)$, it follows Weyl's monotonicity principle [1] and the inequality (2.4) that

$$
s_{j}\left(A_{1}+A_{2}\right) \leq \sqrt{2} s_{j}(A)
$$

for $j=1,2, \cdots$

Inequality (2.5) is equivalent to saying that

$$
\frac{1}{\sqrt{2}} s_{j}\left(A_{1}+A_{2}\right) \leq s_{j}(A)
$$

for $j=1,2, \cdots$

Remark 1. (i) Equality holds in the right hand side of Theorem 2.2 if either $A_{1}=0$ or $A_{1}=0$.

(ii) Equality holds in the left hand side of theorem 2.2 if $A_{1}=A_{2}$.

We will present operator version of Theorem 2.1, inequality (2.2).

Remark 2. Let

$$
X=\left[\begin{array}{cc}
0 & A \\
-A & 0
\end{array}\right]
$$

where $A$ is normal operator. Then $X$ is normal operator with $X=X_{1}+i X_{2}$ is the Cartesian decomposition of $X$. 


$$
\begin{gathered}
X_{1}=\left[\begin{array}{cc}
0 & \frac{A-A^{*}}{2} \\
\frac{A^{*}-A}{2} & 0
\end{array}\right] \text { and } X_{2}=\left[\begin{array}{cc}
0 & \frac{A+A^{*}}{2 i} \\
\frac{-A-A^{*}}{2 i} & 0
\end{array}\right] . \\
\text { It follows that }\left|X_{1}\right|=\left[\begin{array}{cc}
\frac{\left|A^{*}-A\right|}{2} & 0 \\
0 & \frac{\left|A-A^{*}\right|}{2}
\end{array}\right] \text {, and }\left|X_{2}\right|=\left[\begin{array}{cc}
\frac{\left|A+A^{*}\right|}{2} & 0 \\
0 & \frac{\left|A+A^{*}\right|}{2}
\end{array}\right] \text {. }
\end{gathered}
$$

Now, by direct calculations and applying Theorem 2.2 we get

$$
\frac{1}{\sqrt{2}} s_{j}\left(A_{1}-A_{2}\right) \leq s_{j}(A) \leq s_{j}\left(\left|A_{1}\right|+\left|A_{2}\right|\right)
$$

for $j=1,2, \cdots$

Remark 3. We note that the right hand side of the inequality (2.6) is the same as the inequality (1.6), but the left hand side of the inequalities (1.6) and (2.6) says that the singular value of the addition or subtraction of the Cartesian decomposition for the normal operator $A$ divided by $\sqrt{2}$ is less than or equal to the singular value of the normal operator itself.

As an application of the Theorem 2.2, we will determine upper and lower bounds for singular values of the normal operator $A+i A^{*}$, where $A$ is normal.

Theorem 2.3. Let $A \in K(H)$ be normal operator, where $A=A_{1}+i A_{2}$ is the Cartesian decomposition of
A. Then

$$
\sqrt{2} s_{j}\left(A_{1}+A_{2}\right) \leq s_{j}\left(A+i A^{*}\right) \leq 2 s_{j}\left(A_{1}+A_{2}\right)
$$

for $j=1,2, \cdots$

Proof. Note that $T=A+i A^{*}$ is normal operator, so we can write the Cartesian decomposition of $T$ as

$$
T=T_{1}+i T_{2},
$$

where $T_{1}=\frac{\left(A+A^{*}\right)+i\left(A^{*}-A\right)}{2}$, and

$$
T_{2}=\frac{\left(A-A^{*}\right)+i\left(A^{*}+A\right)}{2 i},
$$

where the cartesian decomposition of $A$ is given by $A=A_{1}+i A_{2}$. By making comparison of $T_{1}$ and $T_{2}$ we see easily that $T_{1}=T_{2}$. It follows that $T_{1}+T_{2}=\left(A+A^{*}\right)+i\left(A^{*}-A\right)=2 A_{1}+2 A_{2}$. Moreover,

$$
\begin{aligned}
\left|T_{1}\right| & =\left(\frac{\left(A^{*}+A\right)-i\left(A-A^{*}\right)}{2} \times \frac{\left(A+A^{*}\right)+i\left(A^{*}-A\right)}{2}\right)^{1 / 2}=\left(\frac{A^{*}+A-i A+i A^{*}}{2} \times \frac{A+A^{*}+i A^{*}-i A}{2}\right)^{1 / 2} \\
& =\left(\frac{\left(2 A_{1}+2 A_{2}\right)\left(2 A_{1}+2 A_{2}\right)}{4}\right)^{1 / 2}=\frac{1}{2}\left|2 A_{1}+2 A_{2}\right|=\left|A_{1}+A_{2}\right|
\end{aligned}
$$

Similarly, $\left|T_{2}\right|=\left|A_{1}+A_{2}\right|$. Now, apply Theorem 2.2 to get

$$
\frac{1}{\sqrt{2}} s_{j}\left(2 A_{1}+2 A_{2}\right) \leq s_{j}(T) \leq s_{j}\left(2\left|A_{1}+A_{2}\right|\right)
$$

for $j=1,2, \cdots$ This is equivalent to saying that

$$
\sqrt{2} s_{j}\left(A_{1}+A_{2}\right) \leq s_{j}\left(A+i A^{*}\right) \leq 2 s_{j}\left(A_{1}+A_{2}\right)
$$

for $j=1,2, \cdots$

We will give simple and new proof to the inequality
(1.2).

Theorem 2.4. Let $A, B \in K(H)$ such that $A$ is self-adjoint, $B \geq 0$, and $\pm A \leq B$, then

$$
2 s_{j}(A) \leq s_{j}((B+A) \oplus(B-A))
$$

for $j=1,2, \cdots$

Proof. Since $A$ is self-adjoint operator, we can write $A$ in the form $A=\frac{(B+A)-(B-A)}{2}$, apply the inequality (1.4) we get

$$
s_{j}(A)=\frac{1}{2} s_{j}((B+A)-(B-A)) \leq \frac{1}{2} s_{j}((B+A) \oplus(B-A))
$$

which is equivalent to saying that

$$
2 s_{j}(A) \leq s_{j}((B+A) \oplus(B-A))
$$


for $j=1,2, \cdots$

Audeh and Kittaneh separates Jordan of self-adjoint operator in the inequality (1.3). Here we will give a shorter proof.

Theorem 2.5. Let $A, B \in K(H)$ be self-adjoint operators. Then

$$
s_{j}(A+B) \leq s_{j}\left(\left(A^{+}+B^{+}\right) \oplus\left(A^{-}+B^{-}\right)\right)
$$

for $j=1,2, \cdots$

Proof. Since $A$ and $B$ are self-adjoint operators, we can write $A$ in the form $A=A^{+}-A^{-}$, and similarly we will write $B$ in the form $B=B^{+}-B^{-}$. Apply the inequality (1.4) we get

$$
\begin{aligned}
& s_{j}(A+B)=s_{j}\left(A^{+}-A^{-}+B^{+}-B^{-}\right) \\
s_{j}(A+B) & =s_{j}\left(A^{+}-A^{-}+B^{+}-B^{-}\right) \\
& =s_{j}\left(\left(A^{+}+B^{+}\right)-\left(A^{-}+B^{-}\right)\right) \quad \text { for } j=1,2, \cdots \\
& \leq s_{j}\left(\left(A^{+}+B^{+}\right) \oplus\left(A^{-}+B^{-}\right)\right)
\end{aligned}
$$

We will present the following two theorems as an application to the inequality (1.5).

Theorem 2.6. Let $A \in K(H)$ be self-adjoint operator. Then

$$
s_{j}(A) \leq 2 s_{j}\left(\left(2 A_{1}^{-}+2 A_{2}^{-}\right) \oplus\left(A_{1}+A_{2}\right)\right)
$$

for $j=1,2, \cdots$

Proof. It was proved in Theorem 2.2 that if $A$ is normal operator with Cartesian decomposition

$A=A_{1}+i A_{2}$, then $s_{j}(A) \leq s_{j}\left(\left|A_{1}\right|+\left|A_{2}\right|\right)$ for $j=1,2, \cdots$ from this, it follows that

$$
\begin{aligned}
s_{j}(A) & \leq s_{j}\left(\left(\left|A_{1}\right|-A_{1}\right)+\left(\left|A_{2}\right|-A_{2}\right)+\left(A_{1}+A_{2}\right)\right) \\
& =s_{j}\left(\left(2 A_{1}^{-}+2 A_{2}^{-}\right)+\left(A_{1}+A_{2}\right)\right) \\
& \leq 2 s_{j}\left(\left(2 A_{1}^{-}+2 A_{2}^{-}\right) \oplus\left(A_{1}+A_{2}\right)\right)
\end{aligned}
$$

for $j=1,2, \cdots$

The following theorem is the second application of the inequality (1.5).

Theorem 2.7. Let $A \in K(H)$ be self-adjoint operator. Then

$$
s_{j}\left(A^{+}\right) \leq s_{j}(A \oplus|A|)
$$

for $j=1,2, \cdots$ Moreover,

$$
s_{j}\left(A^{-}\right) \leq s_{j}(A \oplus|A|)
$$

for $j=1,2, \cdots$
Proof. It is well known that $A^{+}=\frac{|A|+A}{2}$, so using
the inequality (1.5) we get the inequality (1.5) we get

$$
s_{j}\left(A^{+}\right)=s_{j}\left(\frac{|A|+A}{2}\right) \leq 2 s_{j}\left(\frac{|A|}{2} \oplus \frac{A}{2}\right)=s_{j}(A \oplus|A|)
$$

for $j=1,2, \cdots$
Similarly, $\quad A^{-}=\frac{|A|-A}{2}, \quad$ so using the inequality (1.5) we get

$s_{j}\left(A^{-}\right)=s_{j}\left(\frac{|A|+(-A)}{2}\right) \leq 2 s_{j}\left(\frac{|A|}{2} \oplus \frac{-A}{2}\right)=s_{j}(A \oplus|A|)$

for $j=1,2, \cdots$

Bhatia and Kittaneh have proved in [6] that if $A, B \in K(H)$, then

$$
s_{j}\left(A B^{*}+B A^{*}\right) \leq s_{j}\left(\left(A^{*} A+B^{*} B\right) \oplus\left(A A^{*}+B B^{*}\right)\right)
$$

for $j=1,2, \cdots$ For related Cauchy-Schwarz type inequalities, we refer to [2] and references therein. Here, we will present similar new inequality.

Theorem 2.8. Let $A, B \in K(H)$ be operators. Then

$$
s_{j}(A B+B A) \leq s_{j}\left(\left(A^{*} A+B^{*} B\right) \oplus\left(A A^{*}+B B^{*}\right)\right)
$$

$\begin{aligned} & \text { for } j=1,2, \cdots \\ & \text { Proof. Suppose } \\ & \text { This implies that }\end{aligned} \quad=\left[\begin{array}{cc}A & 0 \\ B^{*} & 0\end{array}\right]$ and $Y=\left[\begin{array}{cc}B & 0 \\ A^{*} & 0\end{array}\right]$.

$$
X X^{*}=\left[\begin{array}{cc}
A A^{*} & A B \\
B^{*} A^{*} & B^{*} B
\end{array}\right] \text {, and } X^{*} X=\left[\begin{array}{cc}
A^{*} A+B B^{*} & 0 \\
0 & 0
\end{array}\right] \text {. }
$$

On the other hand, we have

$$
Y Y^{*}=\left[\begin{array}{cc}
B B^{*} & B A \\
A^{*} B^{*} & A^{*} A
\end{array}\right] \text {, and } Y^{*} Y=\left[\begin{array}{cc}
B^{*} B+A A^{*} & 0 \\
0 & 0
\end{array}\right] \text {. }
$$

Since $X X^{*}$ and $Y Y^{*}$ are positive operators, then $X X^{*}+Y Y^{*}=\left[\begin{array}{cc}A A^{*}+B B^{*} & A B+B A \\ B^{*} A^{*}+A^{*} B^{*} & B^{*} B+A^{*} A\end{array}\right]$ is positive operator. Now by applying the inequality (1.1), we get

$$
s_{j}(A B+B A) \leq s_{j}\left(\left(A^{*} A+B^{*} B\right) \oplus\left(A A^{*}+B B^{*}\right)\right)
$$

for $j=1,2, \cdots$

\section{REFERENCES}

[1] R. Bhatia, “Matrix Analysis, GTM169,” Springer-Verlag, New York, 1997. http://dx.doi.org/10.1007/978-1-4612-0653-8

[2] I. C. Gohberg and M. G. Krein, "Introduction to the Theory of Linear Nonselfadjoint Operators,” American Mathematical Society, Providence, 1969.

[3] W. Audeh and F. Kittaneh, "Singular Value Inequalities for Compact Operators,” Linear Algebra Applications, Vol. 437, 2012, pp. 2516-2522. http://dx.doi.org/10.1016/j.laa.2012.06.032

[4] X. Zhan, "Singular Values of Differences of Positive Semidefinite Matrices," SIAM Journal on Matrix Analysis and Applications, Vol. 22, No. 3, 2000, pp. 819-823. http://dx.doi.org/10.1137/S0895479800369840

[5] O. Hirzallah and F. Kittaneh, "Inequalities for Sums and 
Direct Sums of Hilbert Space Operators," Linear Algebra Applications, Vol. 424, 2007, pp. 71-82.

http://dx.doi.org/10.1016/j.laa.2006.03.036

[6] R. Bhatia and F. Kittaneh, "The Matrix Arithmetic-Geo- metric Mean Inequality Revisited,” Linear Algebra Applications, Vol. 428, 2008, pp. 2177-2191.

http://dx.doi.org/10.1016/j.laa.2007.11.030 